\title{
Foreign Body (Bullet) in Maxilla
}

\author{
${ }^{1}$ Sandeep Bansal, ${ }^{2}$ Neha Chauhan, ${ }^{3}$ Ashok K Gupta
}

\begin{abstract}
Foreign bodies in the maxillary sinus are not unusual findings. Many cases have been reported so far with foreign bodies like tooth, dental amalgam, chopstick, matchstick, guttapercha point, etc. This paper reports a case of bullet injury with a lodged bullet in maxillary sinus and was retrieved by Caldwell-Luc approach with no intraoperative and postoperative complications.
\end{abstract}

Keywords: Bullet, Foreign body, Maxilla, Removal.

How to cite this article: Bansal S, Chauhan N, Gupta AK. Foreign Body (Bullet) in Maxilla. J Postgrad Med Edu Res 2016;50(1):30-32.

\section{Source of support: Nil}

\section{Conflict of interest: None}

\section{INTRODUCTION}

Most of foreign bodies of maxilla are iatrogenic in nature and most commonly follow dental procedures. Only few cases of non-dental paranasal sinus foreign body have been reported in the literature. External trauma accounts for another important cause. Management of foreign bodies lodged in orbit ad paranasal sinuses varies according to their size, shape, composition, location and wound of entry. ${ }^{1}$

Foreign body in the maxillary antrum may present as an acute phenomenon or may remain silent for years. Some patients present with swelling in the cheek, nasal discharge, nasal regurgitation due to oroantral fistula, etc. to name a few. Computerized tomographic scan aids in diagnosis and should be done in all suspected cases apart from routine radiographic investigations. Some of them masquerade as a neoplastic lesion even. Retrieval of the foreign body is done by either Caldwell-Luc method or minimally invasive endoscopic assisted surgery, both having their own merits and demerits.

\footnotetext{
${ }^{1}$ Assistant Professor, ${ }^{2}$ Senior Resident, ${ }^{3}$ Professor

${ }^{1-3}$ Department of Otolaryngology-Head and Neck Surgery Postgraduate Institute of Medical Education and Research Chandigarh, India
}

Corresponding Author: Sandeep Bansal, Assistant Professor Department of Otolaryngology-Head and Neck Surgery Postgraduate Institute of Medical Education and Research Chandigarh, India, Phone: 911722756760 , e-mail: drsandeepb@ rediffmail.com
Different types of foreign bodies are reported in the maxillary sinus, ranging from bunch of hair and pellets, ${ }^{2}$ pen, ${ }^{3}$ broken spear head, ${ }^{4}$ pieces of wood ${ }^{5}$ and even antral rhinolithiasis around the tooth filling ${ }^{6}$ have been reported earlier. Foreign body reported here further adds to the list.

\section{CASE REPORT}

A 28-year-old male presented to our emergency with a history of bullet injury. On examination, his vitals were within normal limits. Local examination showed a lacerated wound over and above the left side of the lip. The wound measured approximately $3 \mathrm{~cm}$ in its longest dimensions and had irregular margins. It was a deep wound and on palpation blood clots were found within the wound and broken fragments of anterior wall of maxilla could be felt through it. There was mild swelling of the left cheek. Nose examination was normal and vision was within normal limits.

An X-ray nose and paranasal sinuses was done. It showed an irregular elongated radiopaque foreign body in left maxillary sinus (Fig. 1A). The relation of the foreign body with the surrounding structures was seen by getting a computed tomography (Fig. 1B).

Patient was taken up for exploration under general anesthesia. As there was a lacerated wound over the region of canine fossa, Caldwell-Luc approach was used to expose the maxillary sinus. Anterior wall of sinus was fractured and fractured fragments were removed to expose the sinus. Foreign body was visualized lying in the sinus. It was impacted laterally into the lateral maxillary wall. The bullet was carefully disimpacted from the surrounding tissues and was removed. Two small pellets were also present on the floor of the sinus which were removed as well. A betadine roller gauge was kept in the sinus and was brought out of the intraoral defect laterally, suturing the medial part of the wound. The entry wound over the lip was closed after through irrigation of the sinus and achieving hemostasis. Pack was removed on postoperative day 1 and repeat $X$-ray PNS was done (Fig. 2). No foreign body was seen. Patient was discharged on oral antibiotics.

\section{DISCUSSION}

Out of all foreign bodies in the paranasal sinuses, about $50^{5}$ to $75 \%{ }^{7}$ are found in the maxillary sinuses. There 

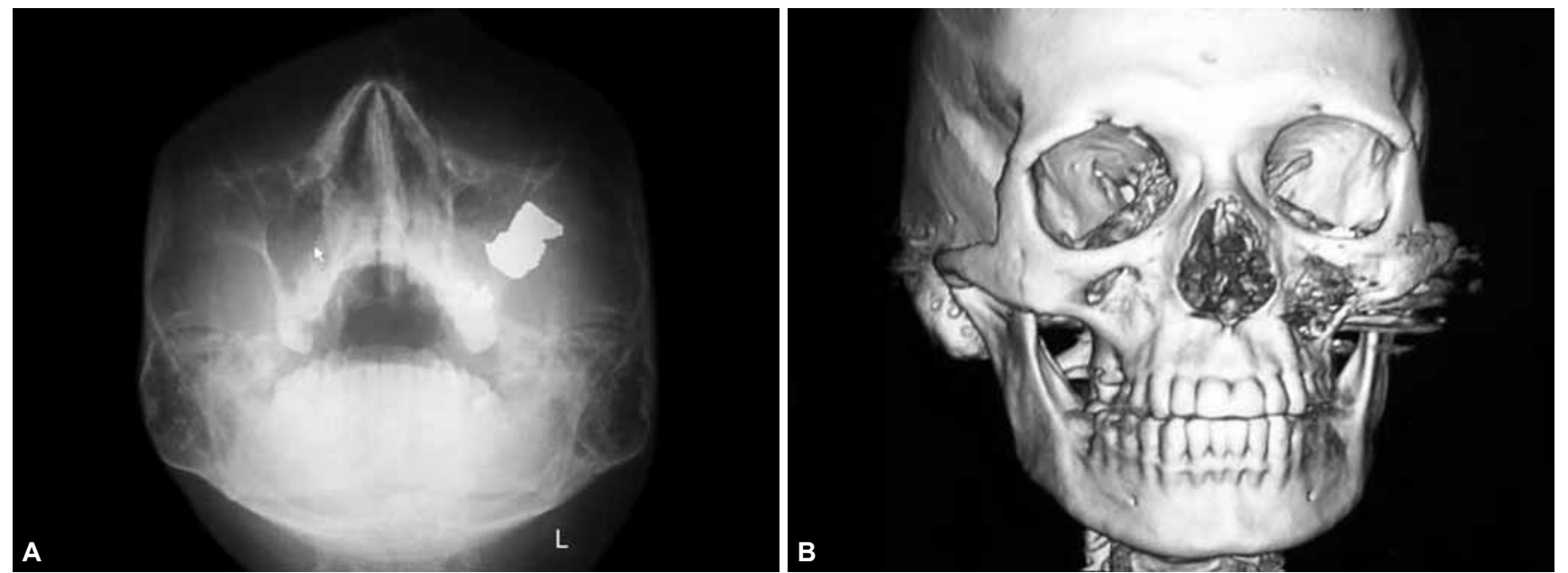

Figs 1A and B: Preoperative X-ray PNS showing presence of radiopaque foreign body in left maxillary antrum directed in the posterolateral direction and (B) 3D reconstruction; fractured anterior and lateral wall with intact orbital floor

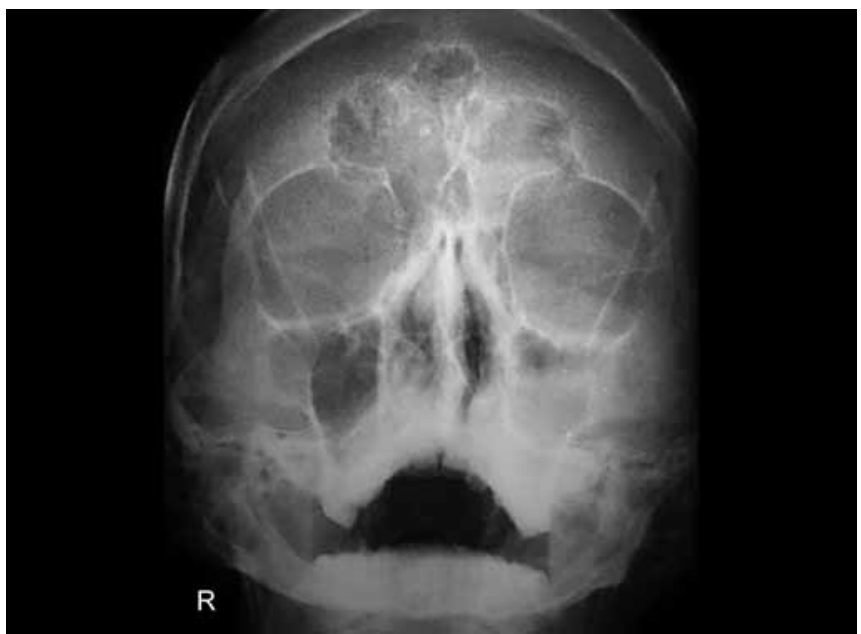

Fig. 2: Postoperative X-ray showing no remnant foreign body and no subcutaneous emphysema

are many instances where tooth, dental amalgams, matchsticks, needles, pellets, etc. have been discovered. Some foreign bodies even remain unnoticed for years $.8,9$ Unusual foreign body like a bunch of hair, following a gunshot injury was also reported in literature. ${ }^{2}$

Patients may have different signs and symptoms or they may remain completely asymptomatic. They may present with cheek swelling, headache, nasal discharge, stuffiness, oroantral fistula or headache. A foreign body may appear to be in different positions inside the sinus. Foreign bodies may initiate inflammation in the antrum involved and can be a nidus for calcification too. It has been suggested that foreign bodies produce chronic physical and chemical irritation of the mucosa. Killey and $\mathrm{Kay}^{10}$ noted that although sinusitis was a common occurrence after entry of a foreign body into the antrum, the onset of change in the antral lining may be delayed for months or even years leading to a degree of ciliary insufficiency and secondary infection.

A routine radiographic investigation points to the diagnosis. When there is no distinction evident in a tomographic scan, magnetic resonance imaging (MRI) can be done or an antroscopy may be performed to confirm a diagnosis.

The removal of the foreign body from the maxillary sinus depends on the size and site of foreign body. For small and medially placed foreign bodies, the most common technique is endoscopic sinonasal surgery allowing the removal via a wide endonasal meatotomy ${ }^{11}$ When extraction is not possible by the endonasal approach, it can be conducted through an external approach by oral antrostomy or a combined approach of endonasal meatotomy and oral antrostomy. ${ }^{12}$ The Caldwell-Luc approach was the gold standard for the access to maxillary sinus for removal of impacted foreign bodies, until the development of endoscopic sinus surgery. Both techniques have limitations and complications. Larger foreign bodies like in our cases require the former approach. In this case, we used the Caldwell-Luc approach as there was an open wound and the bullet was large and impacted on the lateral wall of maxillary sinus.

\section{CONCLUSION}

Maxillary sinus is a common site for foreign body lodgement because of its shape and volume. Removal of a foreign body safely from the paranasal sinus is often a challenging task to the otolaryngologists. Size of the bullet and location of impaction would dictate if it is required to be removed and also helps in deciding the approach to be taken for its removal.

\section{REFERENCES}

1. Tsao YH, Kao CH, Wang HW, Chin SC, Moe KS. Transorbital penetrating injury of paranasal sinuses and anterior skull base by plastic chair glide: management options of a foreign body in multiple anatomic compartments. Otolaryngol Head and Neck Surg 2006;134:177-179. 
2. Sinha V, Sinha AK. Unusual foreign body in the right maxillary antrum (a bunch of hair). Ind J Otolaryngol Head Neck Surg 1989;41(2):64-65.

3. Delgado JA, Bravo G, Nunez JC. A pen inside the maxillary antrum: report of a case. Dentistry 2012;2:5.

4. Gupta AC, Murthy DP, Pulotu ML. Unusual type of foreign body in the maxillary sinus. J Laryngol Otology 1990;104:718-719.

5. Lee D, Nash M, Turk J, Har-El G. Low-velocity gunshot wounds to the paranasal sinuses. Otolaryngol Head Neck Surg 1997 Mar;116(3):372-378.

6. Manjaly G, Pahor AL. Antral rhinolithiasis and tooth filling. Ear Nose Throat J 1994;73(9):676-679.

7. Krause HR, Rustemeyer J, Grunert RR. Foreign body in paranasal sinuses. Mund Kiefer Gesichtschir 2002;6:40-44.
8. Potts BH. Foreign body in the maxillary antrum. Laryngoscope 1905;15(12):939-940.

9. Agarwal RM, Mehrotra AK, Sasibabu K, Mohan V. A metallic foreign body in the maxillary antrum and the nasopharynx. Ind J Otolaryngol Head Neck Surg 1980;32(2):52.

10. Killey HC, Kay LW. Possible sequelae when a tooth or root is dislodged into the maxillary sinus. Br Dent J 1964; 116:73.

11. Tingsgaard PK, Larsen PL. Chronic unilateral maxillary sinusitis caused by foreign bodies in the maxillary sinus. Ugeskr Laeger 1997;159:4402-4404.

12. Friedlich J, Rittenberg BN. Endoscopically assisted CaldwellLuc procedure for removal of a foreign body from the maxillary sinus. J Can Dent Assoc 2005;71(3):200-201. 